\title{
Excipient Innovation Through Precompetitive Research
}

\author{
Yihua Bruce Yu' • Marc B. Taraban' • Katharine T. Briggs' • Robert G. Brinson² • John P. Marino²
}

Received: 29 October 2021 / Accepted: 13 December 2021 / Published online: 20 December 2021

(c) The Author(s), under exclusive licence to Springer Science+Business Media, LLC, part of Springer Nature 2021

KEY WORDS biologics $\cdot$ excipients $\cdot$ formulation $\cdot$ precompetitive research $\cdot$ public-private partnership · vaccines

The mRNA COVID-19 vaccines developed by Pfizer/ BioNTech and Moderna represent a triumph of vaccine development. The core mRNA component of these vaccines is now almost a household name. Unbeknownst to most people, however, is that each of the two mRNA COVID vaccines contains two novel excipients; a cationic lipid and a PEGylated cationic lipid (1, 2), which form the lipid nanoparticle that protects and delivers the mRNA to the patient. Were it not for these two novel excipients, the delivery of mRNA vaccines would be problematic, or not possible at all. This situation is typical for excipients, essential ingredients of pharmaceutical products that work behind the scenes and go unnoticed by the general public. Compared to the development of novel drugs, the development of novel excipients has received much less attention and investment. In this Perspective, we argue that excipients should be treated as infrastructure of the pharmaceutical sector and given their criticality in therapeutic success, there is a need for focused research aimed specifically at novel excipient discovery and development.

Yihua Bruce Yu

byu@rx.umaryland.edu

1 Bio- and Nano-Technology Center, University of Maryland School of Pharmacy, and Institute for Bioscience and Biotechnology Research, Rockville, MD 20850, USA

2 Institute for Bioscience and Biotechnology Research, National Institute of Standards and Technology and the University of Maryland, Rockville, MD 20850, USA

\section{REGULATORY FRAMEWORK FOR EXCIPIENTS}

\section{Active vs Inactive Ingredients in Drug Products}

Pharmaceutical products contain both active and inactive ingredients. In the US, the Code of Federal Regulations (CFR) provides definitions for both. "Active ingredient means any component of a drug product intended to furnish pharmacological activity or other direct effect in the diagnosis, cure, mitigation, treatment, or prevention of disease, or to affect the structure or any function of the body of humans or other animals"(3). The active ingredient is also called active pharmaceutical ingredient (API). "Inactive ingredient means any component of a drug product other than the active ingredient"(3).

\section{Role of Excipients}

The U.S. Food and Drug Administration (FDA) defines excipients as "any inactive ingredients that are added intentionally to therapeutic or diagnostic products, but they are not intended to exert therapeutic effects at the intended dosage, although they may act to improve product delivery" (4). Most, if not all, drug products contain excipients. In a typical drug product, there is one API or sometimes two, but multiple excipients. In terms of total weight, excipients may constitute over $50 \%$ of solid dosage forms and over $90 \%$ of liquid dosage forms.

Excipients are used to overcome limitations of the API, such as low solubility, permeability and stability. 
Functionally, excipients might be solvents, stabilizers, buffers, cryoprotectants, emulsifiers, antimicrobials, etc. Vaccine adjuvants are also regarded as excipients by regulators and industry. Chemically, excipients might be inorganic salts (e.g., $\mathrm{NaCl})$, organic small molecules (e.g., sorbitol), proteins (e.g., human serum albumin), polymers (e.g., polysorbate 80 ), and water for liquid dosage forms.

\section{Regulatory Review of Excipients}

The FDA reviews excipients in the context of drug products, as part of an investigational new drug application (IND), a new drug application (NDA), or a biological license application (BLA). There is no separate review process for excipients at the present time. In other words, the FDA does not review excipients outside the context of IND, NDA or BLA, so the development and use of excipients is tied to new drug products (new APIs or new formulation of existing APIs). The situation is similar in the European Union and Japan.

\section{Recognized Excipients}

Since excipients are reviewed as part of drug products, they receive no standalone approval or licensure. Instead, excipients present in FDA-approved drug products are regarded as recognized by the FDA (5). Industry regards such excipients as allowed or permitted $(6,7)$.

Excipients present in drug products approved through the NDA or ANDA (abbreviated new drug application) pathways are listed in the FDA's Inactive Ingredient Database (IID) (8), while excipients present in vaccines, licensed through the BLA pathway, are listed in Appendix B of the CDC's Pink Book (9). Small molecule excipients listed in CDC's Pink Book are, for the most part, a subset of excipients listed in the FDA's IID, although exceptions exist. For example, $D$-mannose and $L$-asparagine are listed in the Pink Book but not the IID.

Excipients in radiopharmaceuticals may be missing from both the IID and the Pink Book. One example is inositol, an excipient in the radio-diagnostic agent OctreoScan. Also, excipients in non-vaccine biologics licensed through the BLA pathway may be missing from both the IID and the Pink Book. One example is reduced glutathione, which is an excipient in the antihemophilic factor, ADVATE.

\section{Novel vs. New excipients}

The FDA defines a novel excipient as "an excipient that has not been previously used in FDA-approved drug products and that does not have established use in foods" (5).

Whereas, the FDA defines new excipients as excipients "that are not fully qualified by existing safety data with respect to the currently proposed level of exposure, duration of exposure, or route of administration" (10).

The FDA definition of new excipients is broader that of novel excipients, i.e., not every new excipient is a novel excipient. For example, xylitol is not a novel excipient because it is present in FDA-approved drug products and is listed in the IID. However, xylitol has appeared in FDA-approved oral dosage forms, according to the IID. If xylitol is proposed for use in a parenteral drug product-new route of administration-for the US market, it would be considered a new excipient, per the FDA definition.

\section{CHALLENGES AND OPPORTUNITIES FOR EXCIPIENT INNOVATION}

\section{The Need for Novel Excipients}

Novel excipients facilitate drug development and manufacturing and enable new drug products. Without novel excipients, drug development can be hindered or even stalled. In 2019, the United States Pharmacopeia (USP) conducted a novel excipients survey of 264 industry formulation scientists. It was found that when limited to recognized excipients, $84 \%$ of respondents experienced product limitations (e.g., having to use the second-best formulation), $64 \%$ experienced product delays of 1-5 years, and $28 \%$ experienced product development discontinuation (11). From the survey, USP concluded that "the current offerings for excipients permitted for use in US-approved drug products are insufficient to meet the needs of US formulators in drug product development that are critical in facilitating innovation for the advancement of new medical products"(11).

Whereas the two mRNA COVID vaccines are examples of new drug products containing both new APIs and novel excipients, most new drug products contain only a new API without novel excipients. Conversely, a few new drug products contain no new API but novel excipients. One example is the inhalable insulin product Afrezza, which contains an existing API, human insulin, and a novel excipient, fumaryl diketopiperazine (FDKP). FDKP enabled a new dosage form, an inhalable powder, of an existing API. Afrezza was approved by the FDA in 2014 and FDKP is now listed in the IID. Although, traditionally, novel excipients have been developed with a specific API in 
mind, modern medicines would benefit from separate and intensive research to discover and develop novel excipients to support biologics, cell and gene therapy, and other complex drug platforms apart from the API.

The primary driver for novel excipients should be unmet medical needs, such as enabling novel APIs, as exemplified by the two mRNA COVID-19 vaccines, or enabling improved delivery of existing APIs, as exemplified the inhalable insulin product Afrezza. In the area of biologics, an important unmet medical need is the distribution and handling of vaccines and other biologics with less or no reliance on the cold chain (12). Ideally, excipient innovation will be platform agnostic; novel excipients will improve API stability and/or delivery for similar APIs.

\section{Challenges with Excipient Innovation}

The development of novel excipients takes an extremely long timeframe and requires a significant financial investment. Typically, a novel excipient generally takes $6-7$ years to develop, 3-4 years to be incorporated into an approved drug product, and an additional 1-2 years to have a pharmacopeial compendial monograph issued. The whole process takes on average $\sim 12$ years (13). The true cost of this process is hard to specify, as the development of novel excipients and novel APIs are often tied together. In one estimate, the average cost to develop a novel excipient was reported be $\$ 35 \mathrm{M}$ (14). While the cost is high, the return on investment is low and slow. Since excipient sales account for only $0.7 \%$ of worldwide pharmaceutical sales (15), it often takes excipient makers 25 years or more to realize meaningful sales of a novel excipient (16).

Compounding the challenge is the fact that drug makers hesitate to include novel excipients in their products. A study in 2018 found that among drugs listed in the European Public Assessment Report only a few products contain novel excipients (17). This is understandable. Drug products containing novel excipients take a longer time to gain regulatory approval, additional years in some cases. Worse, issues associated with novel excipients, such as a lack of safety data in humans, might cause the drug applications to be rejected (17). Also, makers of generic drugs are disincentivized to use novel excipients because drug products that contain novel excipients are ineligible for the ANDA pathway (5). Overall, drug makers often avoid using novel excipients to minimize regulatory risk; the consequence of avoiding the use of novel excipients could result in developing suboptimal products, or even discontinuation of drug development programs $(11,13)$.

\section{Opportunities in Excipient Innovation}

On December 5, 2019, the FDA published a Federal Register Notice (5), entitled "Novel excipient review program proposal; request for information and comments". The Notice indicates that the FDA is considering a pilot program of a separate review of novel excipients, outside the context of drug products. A separate review of novel excipients will untether excipient development from API development. This Notice has generated support both from industry and the USP (18).

The COVID-19 pandemic also presents an opportunity for developing novel excipients, as evidenced by the novel excipients in the mRNA COVID-19 vaccines. This shows that, when there is a pressing global health need and good prospect of financial return, it is possible to develop novel excipients quickly as may be required for effective new medicines and vaccines.

On Sep. 7, 2021, the FDA launched a voluntary Novel Excipient Review Pilot Program (Pilot Program), soliciting proposals from excipient manufacturers for FDA review of novel excipients prior to their use in drug formulation $(19,20)$. The description of the Pilot Program states that "A novel excipient is any excipient that is not fully supported by existing safety data with respect to the currently proposed level of exposure, duration of exposure, or route of administration." The FDA asks excipient manufacturers in their proposal to describe "the novel excipient, its proposed use, and the public health or drug development need addressed by the excipient." This Pilot Program appears to be the first step in the regulatory pathway toward separate review of novel excipients outside the context of drug products.

\section{PROPOSAL FOR A NEW APPROACH TO EXCIPIENT INNOVATION}

Novel excipients are primarily developed by drug makers for their specific drug products. Since pharmaceutical companies are driven to bring the next product to market, the risk benefit analysis discourages excipient innovation. We therefore propose a new approach to excipient innovation that differs from the current approach: uncoupling excipient development from drug development and stimulating innovation through precompetitive research. 


\section{A Call for Precompetitive Research Focused on Excipient Development}

To date, research in the public domain has also focused on APIs over excipients. As an example, a search of the NIH funding database RePORTER performed on October 29, 2021 found 1,393 projects with "drug" in the title, but only 2 projects with "excipient" in the title. This discrepancy can likely be explained by the nature of API versus novel excipient research. API research intrinsically seeks to treat a known human ailment or disease. It therefore has a specific target outcome and is therefore often of higher profile. Conversely, novel excipient research entails fundamental investigations into the mechanisms whereby molecules enhance API activity and stability. While such work has the ability to benefit the entire industry, it does not necessarily have a higher profile disease target, with the exception when novel excipient development occurs in tandem with an API. But even in such exceptional cases, excipients often get public attention when they are suspected of causing adverse reactions, as with the case of novel excipients in the mRNA COVID-19 vaccines (21).

In addition to facilitating API delivery and handling, a novel excipient must be manufacturable at scale while maintaining good quality, batch-to-batch consistency and meeting acceptable safety profiles. Safety testing is probably the most challenging aspect of novel excipient development. Safety evaluation of excipients in the context of drug products is relatively mature with the procedure outlined in the 2005 FDA guideline (10). However, the safety evaluation of excipients outside the context of drug products is still at the beginning stage. To address this issue, the FDA Pilot Program has included a section on the requirement of toxicology and quality data for the proposed conditions of use (20).

Given all these considerations, research into novel excipients may be better suited for the precompetitive space, where there is not the urgent need to get a drug to market. Furthermore, when novel excipients are developed by drug makers, they are often proprietary and are not accessible by others. In contrast, novel excipients developed through focused precompetitive research and the associated increase in knowledge gained from these studies would more likely become available to the entire community. In a sense, excipients could be regarded as infrastructure for the pharmaceutical sector, like roads and bridges for the transportation sector. Like other infrastructures, precompetitive excipient research may be supported by public-private partnerships.

\section{Developing Novel Excipients for Biologics Through Precompetitive Research}

When drug makers develop novel excipients, it is mostly for a narrow focus and specific products. Instead, by its nature precompetitive research should aim to be as broadly beneficial as possible to the whole pharmaceutical sector. The question is, in the absence of specific APIs, how should precompetitive excipient research proceed?

As excipients for small molecule drugs have a relatively long history and are more mature, we suggest that precompetitive excipient research should focus on biologics, where the need for novel excipients is greater. The fact that most biologics require cold chains for their distribution is a clear indication that there is significant room for excipient innovation in order to stabilize biologics against cold and heat (12). In general, stabilization of APIs by excipients is an important topic. For example, the 2019 USP excipients survey found that the majority of product delays $(57 \%)$ and product discontinuation (52\%) was caused by an inability to sufficiently stabilize the API using recognized excipients (11).

With biologics in mind, we suggest that precompetitive excipient research could have the biggest impact if directed towards mechanistic studies of the functions of excipients in biologics. Excipient selection is often ad hoc, based more on the experience of the formulator and prior usage in foods, cosmetics and other drugs $(13,22)$ than on fundamental understanding of the underlying mechanisms of the function of an excipient. Mechanistic understandings of excipientAPI interactions and excipient-excipient interactions may guide the development of novel excipients as well as the selection of recognized excipients. Mechanistic understanding of how excipients stabilize biologics' APIs (proteins, antibodies, antigens, genes and cells) would involve significant fundamental research in physical chemistry, biophysics and cell biology.

For example, small molecule drugs bind to in vivo proteins such as receptors after injection. The goal of drug discovery research is to identify small molecules that bind tightly to proteins in biological milieu in a specific and stoichiometric fashion. In contrast, excipients bind to pharmaceutical proteins in the formulation before injection. The goal of excipient discovery is to identify small molecules that bind loosely to proteins in formulation media, so that they readily dissociate from proteins once injected, and in a nonspecific and nonstoichiometric fashion, so that sufficient stabilization can be achieved through numerous weak binding. Weak, nonspecific and nonstoichiometric 
binding poses a much greater challenge for biophysical characterization than tight, specific and stoichiometric binding. Hence this line of research may reveal new biophysical insights, which in turn may help us to better understand the role played by weak interactions in biology.

As for identifying potential leads of excipients for biologics, there is a need to go beyond recognized excipients. For stabilizing biologics against heat and cold, osmolytes from extremophiles may be promising. For example, overwintering insects experience large temperature swings during the year with the help of osmolytes $(23,24)$. Once promising excipient candidates are identified by screening or computational modeling, mechanistic studies may follow suit.

Although the approval pathway for novel excipients may vary among different regulatory bodies, the proposed approach is not tied to any specific regulatory framework. Rather, the proposed approach of precompetitive research advanced through public-private partnerships is applicable to excipient innovation globally. If broadly adopted, this could simulate worldwide efforts to address what is a truly a global need for developing novel excipients for improved safety, stability, and/or delivery of vaccines and biotherapeutics.

\section{CONCLUDING REMARK}

Through this Perspective, we hope to make the case for sustained precompetitive innovation in excipient development and stimulate interests in excipient research. We think precompetitive excipient research will likely have its biggest scientific and economic impact on complex and emerging biologics, such as vaccines, gene therapy, and cell therapy products, many of which currently have stability concerns and need for a robust cold chain.

\section{ACKNOWLEDGMENTS AND DISCLOSURES}

These opinions, recommendations, findings, and conclusions do not necessarily reflect the views or policies of NIST or the United States Government. None.

\section{REFERENCES}

1. European Medicines Agency. Assessment Report: Comirnaty Common Name: COVID-19 mRNA vaccine (nucleoside-modified) [Internet]. 2021. p. 1-140. Available from: https:// www.ema.europa.eu/en/documents/assessment-report/ comirnaty-epar-public-assessment-report_en.pdf.

2. European Medicines Agency. Assessment Report: Spikevax (previously COVID-19 Vaccine Moderna) Common Name:
COVID-19 mRNA Vaccine (nucleoside-modified) [Internet]. 2021. p. 1-169. Available from: https://www.ema.europa.eu/ en/documents/assessment-report/spikevax-previously-covid19-vaccine-moderna-epar-public-assessment-report_en.pdf.

3. Title 21 Code of Federal Regulations (CFR) 210.3(b) [Internet]. U.S. Food Drug Adm. Available from: https://www. accessdata.fda.gov/scripts/cdrh/cfdocs/cfCFR/CFRSearch. cfm?fr $=210.3$.

4. FDA Draft Guidance for Industry. Using the Inactive Ingredient Database [Internet]. 2019. Available from: https:// www.fda.gov/regulatory-information/search-fda-guida nce-documents/using-inactive-ingredient-database-guida nce-industry.

5. Novel Excipient Review Program Proposal; Request for Information and Comments. Fed Regist [Internet]. 2019;84 FR 66669-66671. Available from: https://www.federalregister. gov/documents/2019/12/05/2019-26266/novel-excip ient-review-program-proposal-request-for-information-andcomments.

6. Velagaleti R, DeMerlis CC, Brock W, Osterberg R, Goldring JM. Regulatory update: the IPEC novel excipient safety evaluation procedure. Pharm Technol [Internet]. 2009;33:72-82. Available from: https://www.pharmtech.com/view/regul atory-update-ipec-novel-excipient-safety-evaluation-proce dure.

7. Steinberg M, Silverstein I. The Use of Unallowed Excipients. Int J Toxicol [Internet]. SAGE Publications Inc; 2003;22:373-5. Available from: https://doi.org/10.1177/ 109158180302200506

8. FDA Inactive Ingredient Database (Drug Approvals Database) [Internet]. 2020. Available from: https://www.fda. gov/drugs/drug-approvals-and-databases/inactive-ingre dients-database-download.

9. Centers for Disease Control and Prevention. The Pink Book. Appendix B. "Vaccine Excipient and Media Summary" [Internet]. 2020. Available from: https://www.cdc.gov/vacci nes/pubs/pinkbook/downloads/appendices/b/excipienttable-2.pdf.

10. US Food and Drug Administration. Guidance for Industry. Nonclinical Studies for the Safety Evaluation of Pharmaceutical Excipients [Internet]. 2005. p. 1-9. Available from: https:/ / www.fda.gov/regulatory-information/search-fdaguidance-documents/nonclinical-studies-safety-evaluationpharmaceutical-excipients.

11. Sheehan C, Giannone J, Rashed M, Podolsky D, Mitchell T. USP novel excipients survey: Stakeholders' views on the current state of excipient innovation. Pharm Technol. UBM Medica Periodical Publication; 2020. p. 38-43.

12. Yu YB, Briggs KT, Taraban MB, Brinson RG, Marino JP. Grand challenges in pharmaceutical research series: ridding the cold chain for biologics. Pharm Res [Internet]. 2021;38:3-7. Available from: https://doi.org/10.1007/ s11095-021-03008-w.

13. Kolter K, Guth F. Development of New Excipients. In: Koo OMY, editor. Pharm Excipients [Internet]. 1st ed. Hoboken, NJ, USA: John Wiley \& Sons, Inc.; 2016. p. 269-301. Available from: http://doi.wiley.com/10.1002/9781118992432.ch7.

14. Elder DP, Kuentz M, Holm R. Pharmaceutical excipients quality, regulatory and biopharmaceutical considerations. Eur J Pharm Sci [Internet]. 2016;87:88-99. Available from: http://www.sciencedirect.com/science/article/pii/S0928 098715300920.

15. Rayaprolu BM, Strawser JJ, Anyarambhatla G. Excipients in parenteral formulations: selection considerations and effective utilization with small molecules and biologics. Drug Dev Ind Pharm [Internet]. Taylor \& Francis; 2018;44:1565-71. 
Available from: https://doi.org/10.1080/03639045.2018. 1483392.

16. Zawislak, Prescilla; Chokshi R. Driving Excipient Innovation: Overcoming regulatory constraints surrounding novel excipients can elevate the entire industry. Pharm Manuf [Internet]. 2020 Apr 20; Available from: https://www.pharm amanufacturing.com/articles/2020/driving-excipient-innov ation/.

17. Kozarewicz P, Loftsson T. Novel excipients - Regulatory challenges and perspectives - The EU insight. Int J Pharm [Internet]. 2018;546:176-9. Available from: http:/ /www.sciencedir ect.com/science/article/pii/S0378517318303570.

18. Brennan Z. Industry Supports FDA's Plans to Review Novel Excipients Outside of Applications. Regul Aff Prof Soc [Internet]. 2020; Available from: https://www.raps.org/ news-and-articles/news-articles/2020/2/industry-supportsfdas-plans-to-review-novel-exci?feed=Regulatory-Focus.

19. Dexferrum discontinued [Internet]. U.S. Food Drug Adm. Available from: https://www.accessdata.fda.gov/scripts/ cder/daf/index.cfm?event=BasicSearch.process.

20. FDA. Novel Excipient Review Pilot Program [Internet]. Cent. Drug Eval. Res. 2021 [cited 2021 Dec 10]. Available from: https://www.fda.gov/drugs/development-approval-proce ss-drugs / novel-excipient-review-pilot-program.

21. De Vrieze J. Suspicions grow that nanoparticles in Pfizer's COVID-19 vaccine trigger rare allergic reactions. Sci Insid
[Internet]. 2020; Available from: https://www.science.org/ news/2020/12/suspicions-grow-nanoparticles-pfizer-s-covid19-vaccine-trigger-rare-allergic-reactions.

22. Wang W, Ohtake S. Science and art of protein formulation development. Int J Pharm [Internet]. 2019;568:118505. Available from: http://www.sciencedirect.com/science/artic le/pii/S0378517319305472.

23. Koštál V, Zahradníčková H, Šimek P, Zelený J. Multiple component system of sugars and polyols in the overwintering spruce bark beetle, Ips typographus. J Insect Physiol [Internet]. 2007;53:580-6. Available from: http://www.sciencedir ect.com/science/article/pii/S0022191007000558.

24. Walters Jr KR, Pan Q, Serianni AS, Duman JG. Cryoprotectant biosynthesis and the selective accumulation of threitol in the freeze-tolerant Alaskan beetle, Upis ceramboides. J Biol Chem [Internet]. 2009/04/29. American Society for Biochemistry and Molecular Biology; 2009;284:16822-31. Available from: https://pubmed.ncbi.nlm.nih.gov/19403 530.

Publisher's Note Springer Nature remains neutral with regard to jurisdictional claims in published maps and institutional affiliations. 